\title{
Where Art Meets Technology: Integrating Tangible and Intelligent Tools in Creative Processes
}

Janin Koch

Aalto University, Finland

janin.koch@aalto.fi

Jennifer Pearson

Swansea University, UK

j.pearson@swansea.ac.uk

Andrés Lucero

Aalto University, Finland

lucero@acm.org

Miriam Sturdee

Lancaster University, UK

m.sturdee@lancaster.ac.uk
Wendy E. Mackay

Inria, Université Paris-Saclay,

France

mackay@Iri.fr

\section{Makayla Lewis}

University of the Arts London, UK makayla.lewis@arts.ac.uk

\section{Simon Robinson}

Swansea University, UK

s.n.w.robinson@swansea.ac.uk

(c) ACM, 2019. This is the author's version of the work. It is posted here by permission of ACM for your personal use. Not for redistribution. The definitive version was published in CHI '20 Extended Abstracts, April 25-30, 2020, Honolulu, HI, USA.

http://dx.doi.org/10.1145/3334480.3375172

\begin{abstract}
Art and design are essential aspects of our culture and how we interact with the world. Artists and designers use a wide selection of tools, whose impact is rapidly growing with the progression of digital technologies. This change has opened up new opportunities for the $\mathrm{CHI}$ community to build creative supportive tools. The digital switch has come with many benefits such as lowering barriers, mobile work environments and mass production for distribution of work. Along with these benefits we also see challenges for art and design work and its future perception in society. As technology takes a more significant role in supporting art and design what will this mean for the individual artist or designer? The focus of this workshop is to bring together researchers and practitioners to explore what the future of digital art and design will hold. The exploration will centre around synthesizing key challenges and questions, along with ideas for future interaction technologies that consider mobile and tangible aspects of digital art.
\end{abstract}

\section{Author Keywords}

Tangible Interaction, Machine Learning, Art, Design, Creativity 


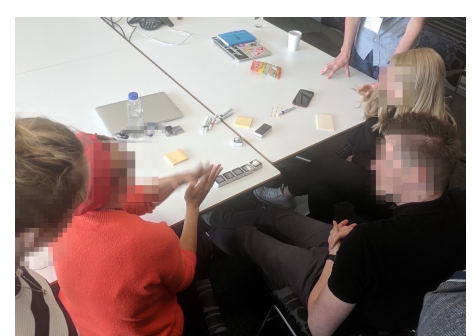

Figure 1: Authors running workshops with designers at BBC

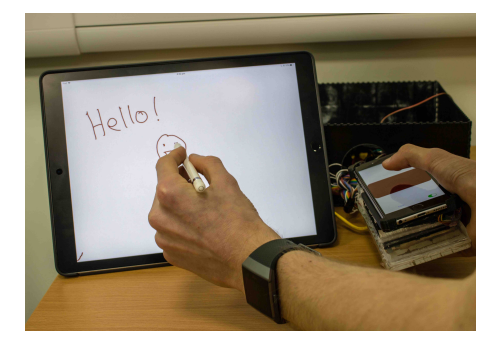

Figure 2: Mobile canvas prototype using hydrogels to simulate paints [14]

\section{CCS Concepts}

-Human-centered computing $\rightarrow$ Human computer interaction $(\mathrm{HCl})$; Interactive systems and tools; Haptic devices; User studies;

\section{Background}

Technology is playing an increasingly significant role in art and design [13]. As there is a growing diversity of tools for creative processes, this workshop aims to bring together people working in these spaces to facilitate discussions and exploration to technology for artists and designers.

An increasing amount of intelligent tools aims to support creative practices in a co-creative manner. Especially in more abstract, tacit areas like design and art, human knowledge can enhance computational processes and vise versa [12]. Machine learning based ideation tools, for example, aim to support the generation of ideas by providing verbal $[1,2,7]$, or visual material [8] to explore creative spaces together with the user by interactively contributing and interpreting inspirational material. This interactivity allows systems to work within the iterative processes which are fundamental to design. However, it can also be used to create more abstract artifacts in art and design, as when machines draw $[3,11]$, improvise dance [5] or improvise music [15] with users.

The ability of machines to contribute own artifacts allows designers and artists to not only be more inspired, but also to use this material to work with and adapt ideas to their own needs and interpretations. We also consider mobile and tangible aspects for deploying intelligent tools. The versatility of mobile devises holds potential for allowing intelligent tools to become an integrated part of the process.

Similarly to this, physical and tangible materials have the ability to carry meaning and can be appropriated in creative support tools. To further enhance this tangible layer we see increased exploration into the possibilities of tangible art and design interfaces. This work aims to combine the benefits of physical tangibility with the freedom of digital tools, as with advantages of digital interactions comes a loss of physical engagement. In some cases these elements borrow from existing traditional methods [6], for example simulating brushes to make advanced styli $[17,16$, 9] or exploring possibilities of tactile sensations and realistic textures, which are usually limited in mobile digital experience $[4,14]$ by appropriating multiple devices as tangible tools (see Figure 2).

The active work in this space demonstrates the strong interest in designing, building, and testing improved tools, and the active commercial participation. Researchers could also take the next step to explore the impact of these developments in practice over long-term use. In this workshop, we will challenge researchers and practitioners to think deeper into how a combination of existing physical and computational tools could yield more usable, interactive and creative tools for the artists and designers. We aim to invite ideation of new physical form factors or digital concepts of art and design tools to understand how we can enhance these experiences.

\section{Goals of the Workshop}

The workshop provides a platform from $\mathrm{HCl}$ researchers and practitioners of different backgrounds to discuss the potential benefits and associated risks of tangible and intelligent technologies in relation to the field of art and design. It aims to create a deeper understanding of designing and evaluating new and existing interaction technologies and their societal implications to creative practices. 
More concretely, our aims with this workshop are to:

- Identify the key opportunities that new interactions and tools can bring to the art and design community;

- Explore contexts and work-flows and discover how such interactions can be deployed;

- Investigate the implications of the use of digital tools on the perception of art and design from the perspectives of the creator and consumer.

- Examine possible directions for prototyping and evaluating new digital and tangible artistic systems.

\section{Organizers}

The workshop organizers are researchers in the area of technology for art and design, focusing on both novel interactions, methodologies and societal impact. The contact person will be Janin Koch (janin.koch@aalto.fi).

Janin Koch is a PhD student at Aalto University focusing on the design and development of collaborative intelligent systems for visual ideation, sensemaking, and reflection in design practice.

Wendy Mackay is a Research Director at Inria and head of the ExSitu research group focused on the design of human-computer partnerships, with an emphasis on interactive tools that support early-stage creative design.

Makayla Lewis is a Postdoctoral Research Fellow at University of the Arts London, she uses mixed methods to explore human factors in private and organization data management. She's also a visual thinker and sketcher, organizing a workshop at DIS2017, courses and special interest groups at $\mathrm{CHI}$, and a multitude of industry conferences.
Jennifer Pearson is a senior lecturer at Swansea University whose research interests span a number of topics including information interaction, co-creation, mobile device interaction, digital reading and $\mathrm{HCl}$ for development.

Andrés Lucero is Associate Professor of Interaction Design at Aalto University. His work focuses on the design and evaluation of novel interaction techniques for mobile devices and other interactive surfaces.

Simon Robinson's research focuses on the human side of mobile interaction, looking beyond the current heads-down and self-focused digital world to include everyone, worldwide, in far-off future design.

Miriam Sturdee is a Research Fellow at Lancaster University, specialising in creative practices in computer science. She holds an MFA in Visual Communication from Edinburgh college of art, and works at the intersection of art and computing, as well investigating how sketching practice can support the development of novel technologies and public engagement.

\section{Pre-Workshop Plan}

Website and Social Media

We will create a website including the call for participants, submission details and contact information under userinterfaces.aalto.fi/WhereArtMeetsTechnology. Post-workshop deadline the website will be an archive of the accepted papers and plan of activities to be conducted during the workshop. We will also integrate a timeline for social media posts using the hashtag (\#Artmeets $\mathrm{HCl}$ ) among social media platforms to encourage a better collection of content. We welcome content from attendees on these channels and wider engagement. We will dedicate one organizer to collating and sharing further information from the workshop as the event progresses. After the 

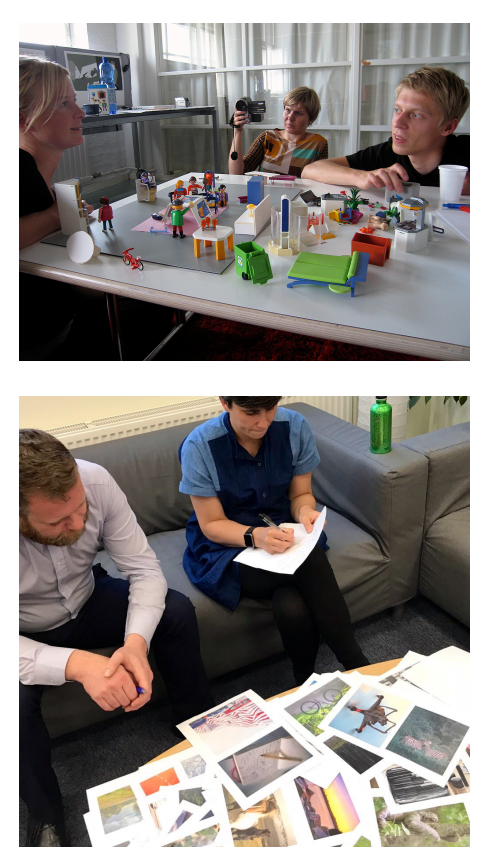

Figure 3: Example of Dialogue-Lab activities from the author's research [10] workshop it will be a platform of discussion for further collaboration and the creation of a public article.

\section{Recruitment}

We intend to recruit a diverse set of scholars and practitioners from the $\mathrm{HCl}$ community and beyond who work with artists and designers, with $\mathrm{Al}$ and Machine learning to assist their processes, or with mobile, tangibles and shape-changing interfaces. We further encourage submissions that address societal views on using such technologies in creative practices. We will recruit via diverse channels e.g. CHI Meta Facebook Group, Twitter, as well as mailing lists of the $\mathrm{HCl}$, Computational Creativity and Design Research Communities. We will reach out to practitioners via Social Media (e.g. IxDA), Industry conferences and meet-ups. We are looking for 15 to 20 participants, who will be asked to submit a 2-4 page positioning paper and will be selected by the quality of the submission and their professional background as well as their contribution to the workshop. The organizers represent a vast amount knowledge and thus will review the submissions.

\section{Required facilities and specifications}

The morning session will require a VGA projector as well as some simple note taking materials (pens and papers). For the rest of the ideation phase and hands-on session the instructors will provide paper prototyping materials and objects for constructing prototypes. More details are described in the workshop plan below. Student volunteers are not required but are very welcome to attend.

\section{Workshop Structure}

This workshop aims to foster and develop future research ideas in the field, along with bringing together like-minded individuals to build a community and encourage collaboration around shared ideas and perspectives.

\section{9:00: Introductions and scene setting from the organizers}

9:10: Icebreaker activity: Each participant spends three-minutes creating a low-fidelity sketch, A5 size or smaller, that act as an introduction, they will then be asked to share their sketch.

09:40 - 11:00: Lightning talks, demo's or presentations: Participants present their submitted papers to the other attendees in form of a lightning talks, demo or presentations (4 min, including max. 2 min Q\&A). In the end of the first session we will introduce prepared scenarios to the attendees.

\section{Mid-morning break}

11:15 11.50: Group discussion: Participants will group around the scenarios based on their personal interest and start discussing potential ideas/concepts and open questions. In the end, each group pitches their ideas to the group (2 $\min$ each).

11:50 12.45: Dialogue-labs: Introduction of an ideation method called dialogue-labs [10] developed by one of the co-authors (A. Lucero) who will guide this session.

Participants will cycle around different stations with inspiring tools and materials (like paper, pens, lego) to aid the generation of new concepts and research questions (Fig. 3).

Lunch: Group lunch offering additional time for discussions and networking.

13:45 15:30: Prototyping activity: Organisers will first summarise the options from the morning session to the group. Afterwards each participant will vote on the ideas 
using provided sticky dot to take forward to prototyping session. We will bring a range of materials and a selection of tools for sketching and building practical resources (e.g. cards, pen, paper, lego). This activity will give the participants the best opportunity to present their ideas in a fun and hands-on way.

\section{Mid-afternoon break}

16:00- 16:45: Presentation of results: Participants will present to the group, and graphic facilitator(s) will be in the room to live record their presentations. Two authors (Lewis and Sturdee) are experienced graphic recorders.

16:45 - 17:30: Closing discussion: Overview of the gathered ideas and their impact on the wider $\mathrm{HCl}$ community, drawing upon the visual and written outputs. Stimulate discussion on how to prototype and evaluate such concepts in general. Discussion of potential publication targets.

\section{End of workshop}

18:00 - open end (optional): Social/networking activities: Continuing discussions in a close by location, which we will reserve beforehand

\section{Planned Outcomes}

Outputs such as specific papers arising from discussions or exercises will be prepared as appropriate. A summary of the collected challenges/ideas around prototyping such tools will be published as a blog post on the workshop website. To reach out to a larger community of practice related to these topics, we further target larger blogging platforms of the UX communities with our results (e.g. Medium). In line with this, we will use real-time documentation (e.g. video streaming, tweets, posts on social media platforms) for a larger audience, e.g. for those unable to join. We also see a potential for a pictorial submission to one of the other ACM conferences such as Creativity\&Cognition, DIS or TEI, which we will discuss in the end of the workshop with the participants. The results of the workshop will be summarized and published on the workshop's website for archival purposes.

\section{Call for Participants}

This $\mathrm{CHI} 2020$ workshop aims to create a deeper understanding of designing and evaluating new and existing interaction technologies, and their societal implications to creative practices. We invite submissions from practitioners and researchers in disciplines such as human factors, computer science, art, and $\mathrm{HCl}$, who are interested in exploring digital technology in art and design practices.

In a one-day workshop, we will start off with lightning talks and demos from accepted participants. We continue with an ideation method called dialogue-labs to generate new ideas for future interaction technologies or evaluation methods for their societal implications on computational creative practices. In the second half of the workshop, participants will build prototypes using pen and paper, Lego, or other materials. The submission should address a topic related to novel tangible and digital technologies for art and design practices. This includes designing/exploring tangible prototypes for digital experiences, creativity support tools for practitioners, and Al/ML-based tools.

Participants are asked to submit 2 to 4-page position papers (in $\mathrm{CHI}$ Extended Abstract PDF format) via the workshop website (userinterfaces.aalto.fi/WhereArtMeetsTechnology) by 11 February 2020 presenting aspects of their own work (or work-in-progress) relevant to the core themes of the workshop, and areas of interest described in the proposal. 
Submissions should also include a short personal biography (max 200 words). These papers will be reviewed by the organizing committee, supported by a wider program committee where necessary.

At least one author of each accepted position paper must register and attend the workshop. For questions, please contact Janin.Koch@aalto.fi

\section{Acknowledgement}

This work has been funded by the European Research Council (ERC) under the European Union's Horizon 2020 research and innovation programme (grant agreement No 637991) and CREATIV: Creating Co-Adaptive HumanComputer Partnerships (grant agreement No 321135).

\section{REFERENCES}

[1] Salvatore Andolina, Khalil Klouche, Diogo Cabral, Tuukka Ruotsalo, and Giulio Jacucci. 2015. InspirationWall: Supporting Idea Generation through Automatic Information Exploration. In Proceedings of the 2015 ACM SIGCHI Conference on Creativity and Cognition. ACM, 103-106.

[2] Patti Bao, Elizabeth Gerber, Darren Gergle, and David Hoffman. 2010. Momentum: Getting and Staying on Topic During a Brainstorm. In Proceedings of the SIGCHI Conference on Human Factors in Computing Systems. ACM, 1233-1236.

[3] Nicholas Davis, Chih-PIn Hsiao, Kunwar Yashraj Singh, Lisa Li, and Brian Magerko. 2016. Empirically studying participatory sense-making in abstract drawing with a co-creative cognitive agent. In Proceedings of the 21st International Conference on Intelligent User Interfaces. ACM.
[4] Yuki Hirobe, Takumi Yoshida, Shinobu Kuroki, Kouta Minamizawa, Katsunari Sato, and Susumu Tachi. 2010. Colorful Touch Palette. In ACM SIGGRAPH 2010 Emerging Technologies (SIGGRAPH '10). ACM, New York, NY, USA, Article 10, 1 pages. DOI : http://dx.doi.org/10.1145/1836821.1836831

[5] Mikhail Jacob, Alexander Zook, and Brian Magerko. 2013. Viewpoints Al: Procedurally Representing and Reasoning about Gestures. In DiGRA Conference.

[6] Daniel F. Keefe, Daniel Acevedo Feliz, Tomer Moscovich, David H. Laidlaw, and Joseph J. LaViola, Jr. 2001. CavePainting: A Fully Immersive 3D Artistic Medium and Interactive Experience. In Proceedings of the 2001 Symposium on Interactive 3D Graphics (I3D '01). ACM, New York, NY, USA, 85-93. DOI : http://dx.doi.org/10.1145/364338.364370

[7] Yui Kita and Jun Rekimoto. 2018. V8 Storming: How Far Should Two Ideas Be?. In Proceedings of the 9th Augmented Human International Conference. ACM, 14.

[8] Janin Koch, Andrés Lucero, Lena Hegemann, and Antti Oulasvirta. 2019. May Al?: Design Ideation with Cooperative Contextual Bandits. In Proceedings of the 2019 CHI Conference on Human Factors in Computing Systems. ACM.

[9] Xin Liu and Jiawei Gu. 2013. FlexStroke: A Flexible, Deformable Brush-tip with Dynamic Stiffness for Digital Input. In Proceedings of the 8th International

Conference on Tangible, Embedded and Embodied Interaction (TEI '14). ACM, New York, NY, USA, 39-40. DOI : http://dx.doi.org/10.1145/2540930.2540982 
[10] Andrés Lucero and Kirsikka Vaajakallio. 2009. Dialogue-labs: creating dialogue in co-design sessions. In Proceedings of the 4th international conference on designing pleasurable products and interfaces. 318-329.

[11] Changhoon Oh, Jungwoo Song, Jinhan Choi, Seonghyeon Kim, Sungwoo Lee, and Bongwon Suh. 2018. I Lead, You Help but Only with Enough Details: Understanding User Experience of Co-Creation with Artificial Intelligence. In Proceedings of the $2018 \mathrm{CHI}$ Conference on Human Factors in Computing Systems. ACM, 649.

[12] Stacey D Scott, Neal Lesh, and Gunnar W Klau. 2002. Investigating human-computer optimization. In Proceedings of the SIGCHI conference on Human factors in computing systems. ACM, 155-162.

[13] Ben Shneiderman. 2007. Creativity Support Tools: Accelerating Discovery and Innovation. Commun. ACM 50, 12 (Dec. 2007), 20-32. DOI : http://dx.doi.org/10.1145/1323688.1323689

[14] Cameron Steer, Simon Robinson, Jen Pearson, Deepak Sahoo, lan Mabbett, and Matt Jones. 2018. A Liquid Tangible Display for Mobile Colour Mixing. In To appear in the proceedings of the 20th International Conference on Human-Computer Interaction with Mobile Devices and Services. ACM, New York, NY, USA.

[15] Belinda Thom. 2000. BoB: an Interactive Improvisational Music Companion. In Proceedings of the fourth international conference on Autonomous agents. ACM, 309-316.

[16] Peter Vandoren, Luc Claesen, Tom Van Laerhoven, Johannes Taelman, Chris Raymaekers, Eddy

Flerackers, and Frank Van Reeth. 2009. FluidPaint: An Interactive Digital Painting System Using Real Wet Brushes. In Proceedings of the ACM International Conference on Interactive Tabletops and Surfaces (ITS '09). ACM, New York, NY, USA, 53-56. DOI : http://dx.doi.org/10.1145/1731903.1731914

[17] Peter Vandoren, Tom Van Laerhoven, Luc Claesen, Johannes Taelman, Fabian Di Fiore, Frank Van Reeth, and Eddy Flerackers. 2008. Dip - It: Digital Infrared Painting on an Interactive Table. In CHI '08 Extended Abstracts on Human Factors in Computing Systems (CHI EA '08). ACM, New York, NY, USA, 2901-2906. DOI : http://dx.doi.org/10.1145/1358628.1358781 